\title{
MULTIBEAM PUMPING OF OPA BY RADIATION OF FIBRE AMPLIFIERS
}

\author{
D. Kezys, M. Gecevičius, A. Piskarskas, V. Pyragaitė, V. Smilgevičius, and A. Stabinis
}

\author{
Laser Research Centre, Vilnius University, Saulètekio 10, LT-10222 Vilnius, Lithuania \\ E-mail: valerijus.smilgevicius@ff.vu.lt
}

Received 22 March 2011; revised 2 June 2011; accepted 21 June 2011

\begin{abstract}
The results of effective optical parametric amplification in KTP crystal pumped by three beams delivered by fibre amplifiers are presented. The second harmonic of three $1.2 \mathrm{~ns}$ and $200 \mu \mathrm{J}$ pulses each extracted from fibre amplifier have been used to pump optical parametric amplifier (OPA) simultaneously. The conversion efficiency of pump radiation into a signal beam was close to $25 \%$.
\end{abstract}

Keywords: nonlinear optics, fibres, erbium, parametric oscillators and amplifiers

PACS: 42.65.-k, 42.55.Wd, 42.65.Yj

\section{Introduction}

The optical parametric amplifiers (OPA) are widely used in many practical applications. The pulsed radiation in nano-, pico-, and femtosecond light scale can be amplified in these devices. In future an amplification of extremely short light pulses up to 10-100 TW power is expected by use of chirped pulse parametric amplification technique (OPCPA [1]). Now as OPCPA pump sources for amplification of fs pulses up to TW power the solid-state flash-lamp pumped amplifiers are used. Although the flash-lamp-pumped laser systems allow to reach few joules pulse energy, the repetition rate of such systems is limited to $10-30 \mathrm{~Hz}$. On the other hand, the diode-pumped solid-state amplifiers let one to achieve high repetition rate (to a few $\mathrm{MHz}$ ), but output energy of these systems are now limited to $1-10 \mathrm{~mJ}$.

The multibeam pump can increase OPA output average power and combine energy from a few low energy $(\sim 1-2 \mathrm{~mJ})$ pump beams to a single signal beam. Firstly, the multibeam pump technique was proposed for travelling-wave optical parametric generator (OPG) [2-4] and later developed for OPA [5-8]. The radiation from laser amplifier divided by beamsplitters into 2 [8] or 3 [5] pump beams was used as OPA pump. In both cases the pump beams were fully coherent. $10 \mathrm{fs}$ duration chirped pulse amplification was demonstrated [7] by using the radiation of three flash-lamp-pumped Nd:YAG amplifiers as a pump. In this case the com- bining of the pump beams into parametrically amplified signal was realized. Another positive aspect of OPA multibeam pump technique is that the parametric amplification does not depend on the phases of pump beams. Moreover, an axial signal beam was nearly diffraction limited in travelling-wave OPG and OPA pumped by spatially incoherent beam [4]. A similar result for OPA was also demonstrated in [9]. We note that parametric diffraction can decrease an efficiency of OPA pumped by several beams. This phenomenon was observed in [7] and investigated in detail in [10]. To avoid the parametric diffraction it is needed to select the intersecting angles of pump beams in OPA crystal volume and to choose properly the pump beam intensity.

One of the possible pump sources for OPCPA could be the amplifiers based on the doped fibres. Recent developments of rare-earth-doped fibre manufacturing permit to create the doubly cladding large-mode-area fibres for lasers and amplifiers and allow one to reach in a single fibre amplifier $\sim 1-2 \mathrm{~mJ}$ pulse energy for $1 \mathrm{~ns}$ pulses [11] with beam quality parameter $M^{2}$ close to 1 . The use of fibre amplifiers for pump of OPA is preferable because in fibre amplifiers there is no limitation for repetition rate up to $1-10 \mathrm{MHz}$ [12].

Further we present the results of an investigation of parametric amplification in type-II KTP crystal pumped by the radiation of three fibre amplifiers. The conversion efficiency into the signal beam in OPA pumped by radiation of three synchronized in time fibre amplifiers 
reached $25 \%$, and such technique could be of prospective for use in optical parametric amplifiers.

\section{OPA multibeam pump geometry}

The main reason to select KTP crystal for multibeam pumped OPA was its high nonlinearity. The multibeam pumped OPA geometry for amplification of $1.064 \mu \mathrm{m}$ wavelength signal with $0.532 \mu \mathrm{m}$ pump was analysed theoretically by numerical calculations of phase-matching condition for $x y$-cut KTP crystal. For a biaxial crystal as KTP, there are three different eigenvalues of the refractive index tensor: $n_{x}, n_{y}$, and $n_{z}$. The possible propagation directions of the pump beams for a given propagation direction of the signal beam were calculated by use of phase matching conditions and Fresnel equations for biaxial crystals. There are three Fresnel equations corresponding to signal, idler, and pump beams. Further, there are two equations corresponding to the transverse phase matching and one equation of the longitudinal phase matching. So, there are six equations to be solved, and six unknown variables: two propagation angles for each wave, $\theta_{j}$ and $\varphi_{j}, j=\mathrm{s}, \mathrm{i}, \mathrm{p}$, where $\mathrm{s}, \mathrm{i}$, and $\mathrm{p}$ denote signal, idler and pump wave, respectively. In our case $\omega_{\mathrm{s}}=\omega_{\mathrm{i}}\left(\omega_{j}\right.$ is a cyclic frequency). We assume that the wave vector of the ordinary signal wave lies in the $x y$ plane, i. e. the angle with the $z$ axis is $\theta_{\mathrm{s}}=90^{\circ}$. When the wave vectors of all three (signal, idler, and pump) waves lie in the $x y$ plane $\left(\theta_{\mathrm{s}}=\theta_{\mathrm{i}}=\theta_{\mathrm{p}}=90^{\circ}\right)$, idler and pump beams are extraordinary. In this case the dependence of the pump propagation angle $\varphi_{\mathrm{p}}$ on the signal angle $\varphi_{\mathrm{s}}$ at exact phase matching is shown in Fig. 1(a). The Sellmeier equations from [13] were adopted. It can be seen that there is no phase matching when $\varphi_{\mathrm{s}}<24.7^{\circ}$. The propagation angles $\varphi_{\mathrm{s}}=\varphi_{\mathrm{p}}=24.7^{\circ}$ correspond to the collinear interaction. When $\varphi_{\mathrm{s}}>24.7$, the noncollinear interaction is possible, and two values of $\varphi_{\mathrm{p}}$ for each $\varphi_{\mathrm{s}}$ were obtained. It means that in this case only two pump beams with wavevectors situated in $x y$ plane can be exactly phase-matched with the same signal beam. In order to employ more pump beams, we have to analyse the interaction outside the $x y$ plane. We assume that propagation angles of the signal beam are $\theta_{\mathrm{s}}=90^{\circ}, \varphi_{\mathrm{s}}=25^{\circ}$ and it is an ordinary beam. Then the possible propagation angles of pump as well as idler beams have been calculated for exact phase-matching, and the results are shown in Fig. 1(b). It is clearly seen that a large number of pump beams can be simultaneously phase-matched with a single signal beam. The possible values of propagation angles $\theta, \varphi$ of pump as

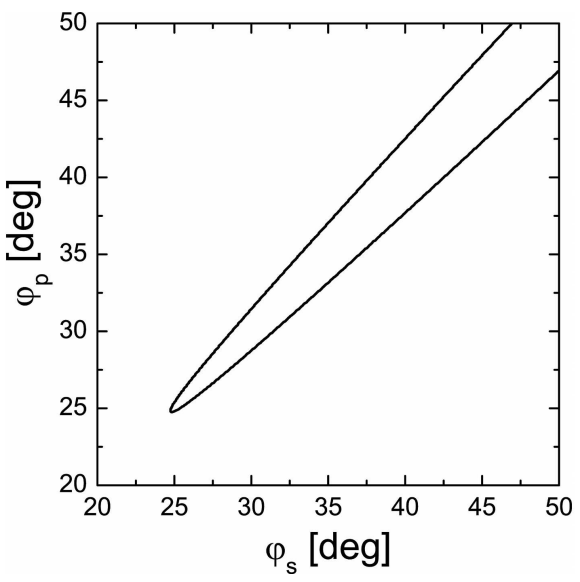

(a)

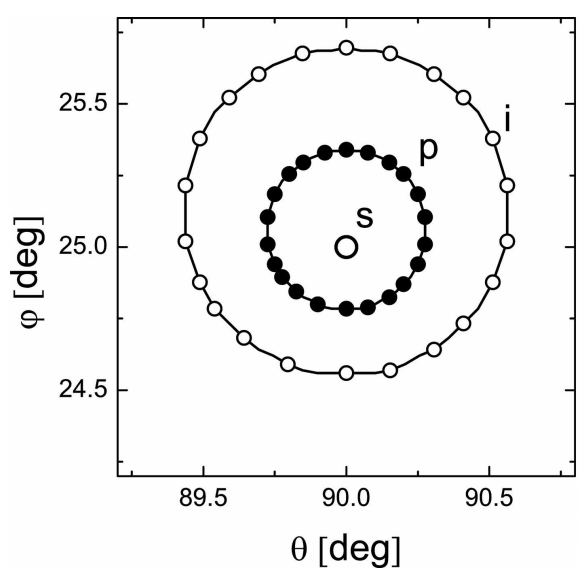

(b)

Fig. 1. (a) Dependence of angle $\varphi_{\mathrm{p}}$ on $\varphi_{\mathrm{s}}$ in KTP crystal. The wave vectors of signal and two pump interacting waves lie in the $x y$ plane. (b) Possible propagation angles of the pump (p) and idler (i) beams when propagation angles of signal (s) beam are $\varphi_{\mathrm{s}}=25^{\circ}, \theta_{\mathrm{s}}=90^{\circ}$.

well as idler beams compose the circumference, and the conical beam also can be used for effective amplification of signal beam [15].

\section{Output energy of OPA. Numerical simulation}

Further, by using the monochromatic plane wave approximation we calculate the energy of signal beam at the output of OPA for different input energy of three pump beams by means of the nonlinear coupled equations:

$$
\begin{aligned}
\frac{\partial A_{\mathrm{s}}}{\partial z} & =\sigma A_{\mathrm{i} 1}^{*} A_{\mathrm{p} 1}+\sigma A_{\mathrm{i} 2}^{*} A_{\mathrm{p} 2}+\sigma A_{\mathrm{i} 3}^{*} A_{\mathrm{p} 3} \mathrm{e}^{\mathrm{i} \Delta z}, \\
\frac{\partial A_{\mathrm{i} 1}}{\partial z} & =\sigma A_{\mathrm{s}}^{*} A_{\mathrm{p} 1}, \\
\frac{\partial A_{\mathrm{i} 2}}{\partial z} & =\sigma A_{\mathrm{s}}^{*} A_{\mathrm{p} 2},
\end{aligned}
$$




$$
\begin{aligned}
& \frac{\partial A_{\mathrm{i} 3}}{\partial z}=\sigma A_{\mathrm{s}}^{*} A_{\mathrm{p} 3} \mathrm{e}^{\mathrm{i} \Delta z}, \\
& \frac{\partial A_{\mathrm{p} 1}}{\partial z}=-2 \sigma A_{\mathrm{s}} A_{\mathrm{i} 1}, \\
& \frac{\partial A_{\mathrm{p} 2}}{\partial z}=-2 \sigma A_{\mathrm{s}} A_{\mathrm{i} 2}, \\
& \frac{\partial A_{\mathrm{p} 3}}{\partial z}=-2 \sigma A_{\mathrm{s}} A_{\mathrm{i} 3} \mathrm{e}^{-\mathrm{i} \Delta z},
\end{aligned}
$$

where $A_{\mathrm{s}}$ denotes the complex amplitude of the signal beam, $A_{\mathrm{i} j}$ and $A_{\mathrm{p} j}$ are the complex amplitudes of the idler and pump beams, respectively, $j=1,2,3$ denotes the number of the beam. $\sigma=\chi^{(2)} \omega_{\mathrm{p}}^{2} /\left(2 c^{2} k_{\mathrm{p}}\right)$ is a coupling coefficient (here $\chi^{(2)}=3.58 \mathrm{pm} / \mathrm{V}$ characterizes the effective quadratic susceptibility of a KTP crystal obtained from Select Non-Linear Optics Software). $c$ is a speed of light, $k_{\mathrm{p}}$ and $\omega_{\mathrm{p}}$ are the wave number and cyclic frequency of the pump beam, respectively, and $\Delta$ is a possible phase mismatch of the third pump beam. Equations (1-7) were simulated in the cases of one, two, and three input pump beams. The results are presented in Fig. 2. When only one input pump beam was present, the amplitude $A_{\mathrm{p} 1}$ was varied from 0 to $A_{\max }$. $A_{\max }$ corresponds to the pulse energy of $100 \mu \mathrm{J}$ and nonlinear length $L_{n}=1 /\left(\sigma A_{\max }\right)$ equal to $0.28 \mathrm{~cm}$. In the case of two input pump beams the first pump beam amplitude $A_{\mathrm{p} 1}$ was fixed at $A_{\max }$, and the second $A_{\mathrm{p} 2}$ was varied from 0 to $A_{\max }$. Further, as the third pump beam was added, the first and the second amplitudes $A_{\mathrm{p} 1}$ and $A_{\mathrm{p} 2}$ were fixed at $A_{\mathrm{max}}$, and $A_{\mathrm{p} 3}$ was varied from 0 to $A_{\max }$. The input amplitude of the signal beam was equal to $A_{\max } / 10$. The input signal as well as pump beams were assumed to be Gaussian with the same beam radius $(90 \mu \mathrm{m})$ and pulse duration (1.2 ns). In this case the beam diffraction and walk-off of pump and idler beams in $1 \mathrm{~cm}$ long KTP crystal can be neglected. The energy of the beams was calculated by integration of corresponding intensity over the area and the time.

We note that the possible phase mismatch of the pump beams $(\Delta \neq 0)$ can provide a better conversion efficiency when compared to the case of the exact phase matching $(\Delta=0)$. As an example, that is demonstrated in Fig. 2 for the case of phase-mismatch of third pump beam. The saturation of an amplification and back-conversion in OPA are the processes which cause a smaller efficiency. In this case the proper phasemismatch can reduce the influence of these processes. For this reason the alignment of all pump beams was

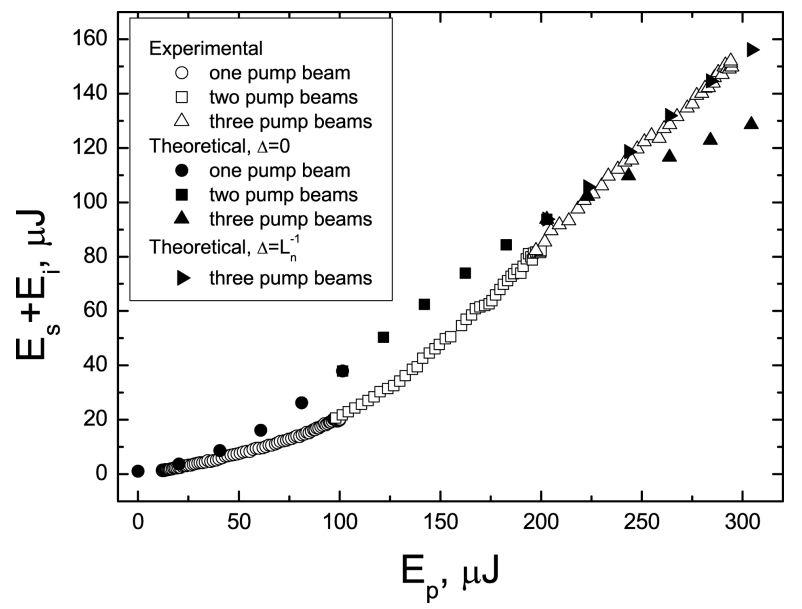

Fig. 2. Theoretical as well as experimental dependence of the output (signal+idler) energy on the input pump energy in OPA.

carried out so that the better efficiency would be obtained in the experiment.

\section{Experiment}

\subsection{OPA pump system}

The experimental set-up of OPA pumped by three beams is presented in Fig. 3. The amplifier system consists of three fibre amplifiers seeded by the Nd:YAG $1.2 \mathrm{~ns}$ duration microchip laser radiation (STANDA LTD product STA-01-5). The seed with efficiency of $30 \%(\sim 5 \mu \mathrm{J})$ was coupled into $3 \mathrm{~m}$ long, $30 \mu \mathrm{m}$ core double-clad NUFERN Yb-doped fibres (PLMAYDF-30/400). The microchip laser was optically isolated from fibre amplifiers by Faraday isolator. The fibres were backward pumped by the fibre-coupled diode lasers (power $\sim 8.8 \mathrm{~W}$ ). For higher mode discrimination the fibres were rolled-up into $7.5 \mathrm{~cm}$ radius bobbin and thermo-stabilized at $30^{\circ} \mathrm{C}$ temperature with computer controlled oven. The intensity distribution of amplified signal beam was close to diffraction-limited (beam quality parameters were $M_{x}^{2}=1.05, M_{y}^{2}=1.10$ ). The maximum output energy of the fibre amplifier was

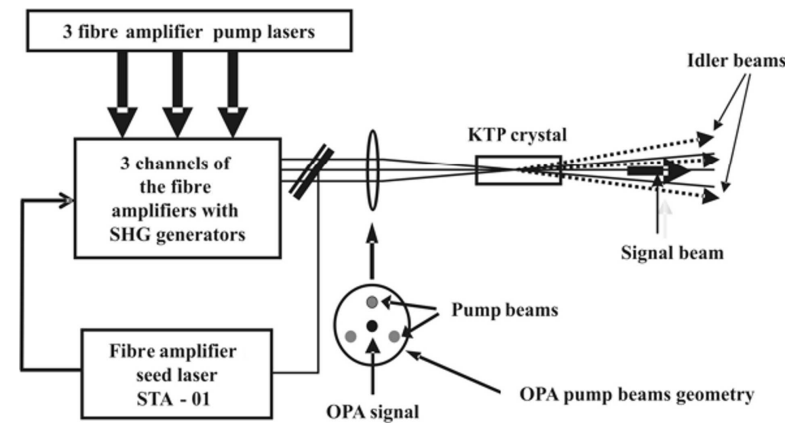

Fig. 3. Experimental set-up. 
$\sim 200 \mu \mathrm{J}$ and was limited by self-phase modulation and stimulated Raman scattering. The spectrum of the amplified radiation was broadened from $\sim 0.6 \mathrm{GHz}$ (seed spectrum) to $\sim 8 \mathrm{GHz}$ for $200 \mu \mathrm{J}$ amplified pulses, being in a good agreement with the results presented in [11]. The second harmonic of amplified radiation (OPA pump) was generated in $6 \mathrm{~mm}$ long KTP crystals with $50 \%$ efficiency.

\subsection{KTP OPA}

The second harmonic radiation of three OPA pump beams was focused into OPA crystal by $750 \mathrm{~mm}$ focal length lens. The intersection angles of the pump beams were $\sim 20 \mathrm{mrad}$. We note that an alignment of angles $\theta$ and $\varphi$ of the pump beams was provided in a such way that an output (signal+idler) energy of OPA should be at maximum. In this case the certain phase-mismatch of three pump beams has been realized. An intensity distribution of pump radiation in OPA KTP crystal volume is presented in Fig. 4 and looks like characteristic interference pattern. The total beam width was $180 \mu \mathrm{m}$. Due to self-phase modulation in the fibre amplifier the interference pattern visibility was $\sim 0.2$, Fig. $4(\mathrm{~b})$. In the case of negligible amplification factor in fibre amplifier $(\approx 2)$ the interference pattern visibility was $\sim 0.93$, Fig. 4(a). As an OPA, $10 \mathrm{~mm}$ long $x y$-cut KTP crystal was used. We tested parametric amplification by seeding into OPA the fundamental radiation of microchip laser (1064 nm). An energy of seed radiation was varied from 1 to $5 \mu \mathrm{J}$. The pump beams and OPA seed signal were overlapped in time and space in the volume of KTP crystal by means of mirrors and optical delay lines.

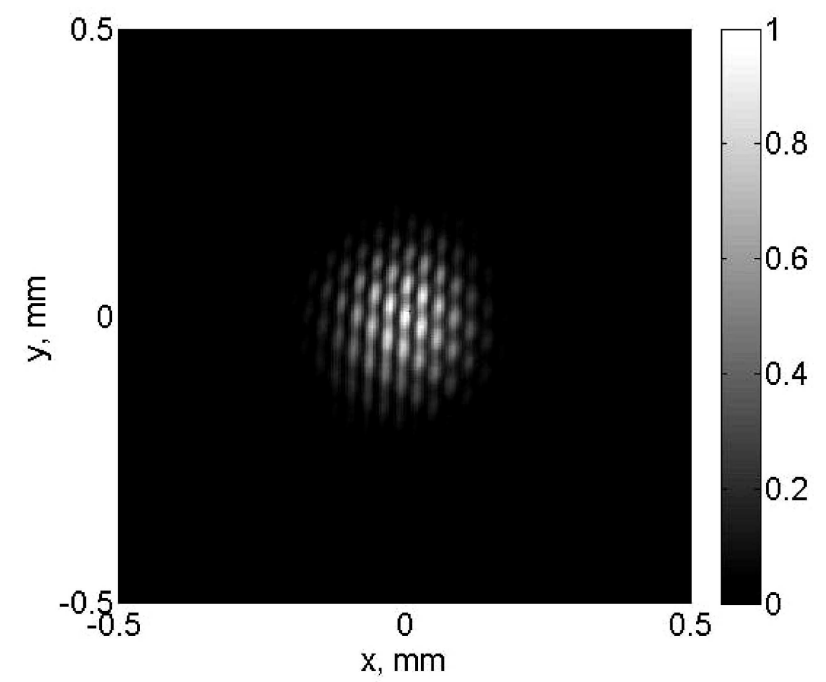

(a)
The seed signal from the microchip laser was injected into OPA at $\sim 10 \mathrm{mrad}$ with respect to pump beam propagation directions as shown in Fig. 3 .

\section{Results and discussion}

First of all, the parametric amplification in the field of single pump beam was investigated. An injected signal beam was orthogonally polarized versus pump beam. The angular spectrum observed when OPA was pumped by single beam is shown in Fig. 5(a). In Fig. 5(b,c) an angular spectrum of signal and idler beams when OPA has been pumped by two and three beams is presented, respectively. In all cases at the output of OPA the signal beam was close to diffraction limited $\left(M^{2} \sim 1\right)$.

We note that parametric amplification of injected signal beam in OPA could be of $10^{6}-10^{7}$ orders. In this case for very high pump intensities together with injected signal an amplification of the parametric superfluorescence radiation will take place. As a result, the decrease of the contrast of the amplified signal occurs. For effective energy conversion it is necessary to inject into OPA the signal with energy of an order $0.01-0.1$ of pump energy [14]. The dependence of OPA output (signal+idler) energy on pump energy is presented in Fig. 2. When OPA was pumped by two beams, the energy of the second beam was gradually increased up to $100 \mu \mathrm{J}$. In this case the maximum energy of amplified signal was $\sim 4$ times higher in comparison with maximum signal energy for single pump case. When OPA was pumped by three beams, the maximum OPA output

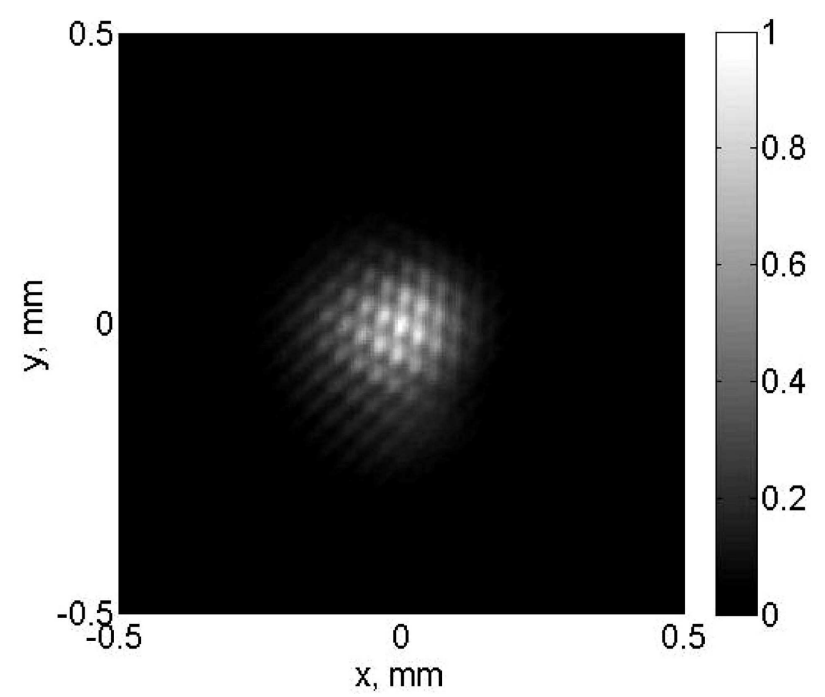

(b)

Fig. 4. Intensity distribution of three pump beams passed through fibre amplifiers after intersection in KTP crystal: (a) passed through fibre with negligible amplification, (b) with maximum amplification in fibre. 


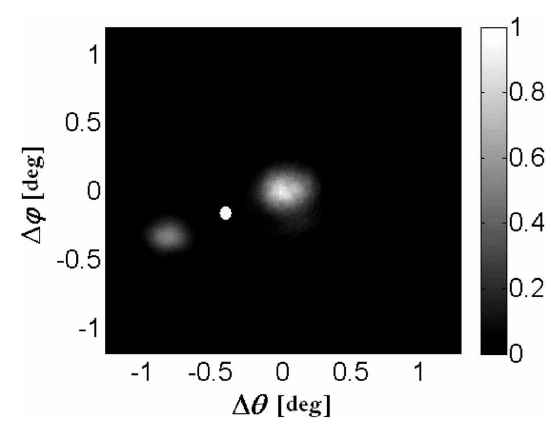

(a)

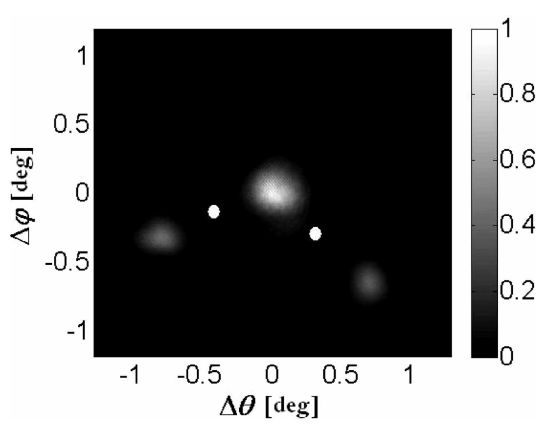

(b)

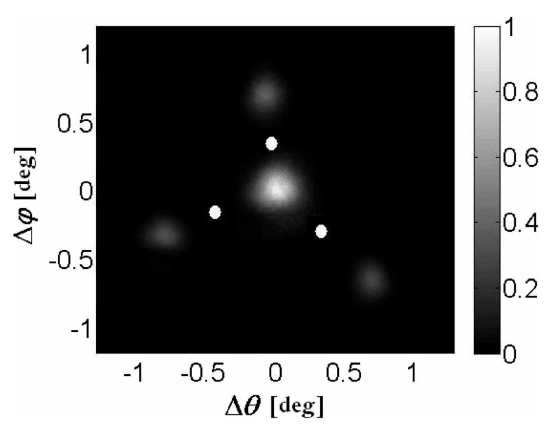

(c)

Fig. 5. Angular spectra of amplified signal and idler beams when KTP OPA was pumped by (a) one, (b) two, and (c) three beams. Filled circles show the positions of the pump beams.

energy was $\sim 150 \mu \mathrm{J}$ and $\sim 25 \%$ energy conversion efficiency was realized. We note that in this case a rather good agreement with the results of numerical simulation (Fig. 2) was obtained. It should be pointed out, that when OPA was pumped by three beams, the pump parametric self-diffraction was also observed, but the efficiency of parametric diffraction was lower than $1 \%$ when compared to maximum pump energy $(\sim 300 \mu \mathrm{J})$.

\section{Conclusion}

The progress in the rare-earth-doped fibre technology together with demonstrated above possibilities of OPA multibeam pump geometry allow one to develop a new class of coherent light sources. An imperfection of pulsed fibre amplifier is its self-phase modulation, which increases amplified signal bandwidth and prevents the formation of high coherence radiation. In contrast, an OPA pumped by fibre amplifier radiation can produce high coherence radiation because the pump beam incoherence is transferred to idler beam. On the other hand, the multibeam pump technique allows one to increase output energy of OPA by combining energy from multiple pump beams. Our experimental research demonstrates an efficient energy conversion ( signal + idler $\sim 50 \%$ ) of pump radiation from three different sources into OPA output. The proposed OPA pumped by the output radiation of three fibre amplifiers opens up new possibilities for combining the energy from a large number of fibre amplifiers.

\section{Acknowledgements}

This work was partially supported by the Research Council of Lithuania (project "INTEGRA"), by LaserLAb Europe HAPPIE JRA, and is also a part of national ELI-oriented activities.

\section{References}

[1] A. Dubietis, G. Jonušauskas, and A. Piskarskas, Powerful femtosecond pulse generation by chirped and stretched pulse parametrical amplification in BBO crystal, Opt. Commun. 88(4-6), 437-440 (1992).

[2] E. Gaizhauskas, A. Piskarskas, V. Smilgyavichyus, and G. Shlekys, Parametric oscillation in $\mathrm{LiNbO}_{3}$ crystal in the presence of a photorefractive diffraction grating, Sov. J. Quantum Electron. 21(5), 577-578 (1991).

[3] V. Smilgevičius and A. Stabinis, Two-beam pumped travelling-wave optical parametric generator, Opt. Commun. 106(1-3), 69-74 (1994).

[4] A. Piskarskas, V. Smilgevicius, A. Stabinis, and V. Vaicaitis, Spatially cumulative phenomena and output patterns in optical parametric oscillators and generators pumped by conical beams, J. Opt. Soc. Am. B 16(9), 1566-1578 (1999).

[5] A. Dubietis, R. Danielius, G. Tamošauskas, and A. Piskarskas, Combining effect in a multiple-beampumped optical parametric amplifier, J. Opt. Soc. Am. B 15(3), 1135-1139 (1998).

[6] G. Tamošauskas, A. Dubietis, G. Valiulis, and A. Piskarskas, Optical parametric amplifier pumped by two mutually incoherent laser beams, Appl. Phys. B 91(2), 305-307 (2008).

[7] S. Ališauskas, R. Butkus, V. Pyragaitè, V. Smilgevičius, A. Stabinis, and A. Piskarskas, Prospects for increasing average power of optical parametric chirped pulse amplifiers via multi-beam pumping, Opt. Commun. 283(3), 469-473 (2010).

[8] D. Herrmann, R. Tautz, F. Travella, F. Krausz, and L. Veisz, Investigation of two-beam-pumped noncollinear optical parametric chirped-pulse amplification for the generation of few-cycle light pulses, Opt. Express 18(5), 4170-4183 (2010).

[9] T. Kurita, K. Sueda, K. Tsubakimoto, and N. Miyanaga, Experimental demonstration of spatially coherent beam combining using optical parametric amplification, Opt. Express 18(14), 14541-14546 (2010).

[10] R. Danielius, P. Di Trapani, A. Dubietis, A. Piskarskas, D. Podenas, and G.P. Banfi, Self-diffraction through 
cascaded second-order frequency-mixing effects in Beta-barium borate, Opt. Lett. 18(8), 574-576 (1993).

[11] C.D. Brooks and F. Di Teodoro, Multimegawatt peakpower, single-transverse-mode operation of a $100 \mu \mathrm{m}$ core diameter, Yb-doped rodlike photonic crystal fiber amplifier, Appl. Phys. Lett. 89(11), 111-119 (2006).

[12] G.T. Moore, C.A. Denman, and T.S. Ross, Using multiple mutually incoherent fiber lasers to pump a coherent signal beam in an optical parametric oscillator, IEEE J. Quantum Electron. 36(7), 828-834 (2000).
[13] D.N. Nikogosyan, Nonlinear Optical Crystals: A Complete Survey (Springer, 2005).

[14] A. Piskarskas, V. Smilgevičius, and J. Jasevičiūtè, Optimization of three-photon parametric excitation of light in the picosecond pulse range, Sov. Phys. Collect. 20(1), 66-74 (1980).

[15] R. Gadonas, A. Marcinkevičius, A. Piskarskas, V. Smilgevičius, and A. Stabinis, Travelling wave optical parametric generator pumped by a conical beam, Opt. Commun. 146(1-6), 253-256 (1998).

\title{
DAUGIAPLUOŠTIS OPTINIO PARAMETRINIO STIPRINTUVO KAUPINIMAS ŠVIESOLAIDINIŲ STIPRINTUVU SPINDULIUOTE
}

\author{
D. Kezys, M. Gecevičius, A. Piskarskas, V. Pyragaitė, V. Smilgevičius, A. Stabinis
}

Vilniaus universitetas, Vilnius, Lietuva

\section{Santrauka}

Pateikti efektyvaus optinio parametrinio stiprinimo KTP kristale, kaupinamo trimis sustiprintais šviesolaidiniuose stiprintuvuose pluoštais, tyrimo rezultatai. Vienalaikiam optinio paramet- rinio stiprintuvo kaupinimui buvo panaudota sustiprintų šviesolaidiniuose stiprintuvuose triju 1,2 ns trukmès ir $200 \mu \mathrm{J}$ energijos impulsų antrosios harmonikos spinduliuotè. Kaupinimo spinduliuotès keitimo efektyvumas ị signalinę bangą siekẻ 25\%. 